\title{
Aplication of Hybrid Method for Superior cattle selection using Decision Support System
}

\author{
L Sumaryanti $^{1 *}$, Nurcholis ${ }^{2}$, and Lusia Lamalewa ${ }^{1}$ \\ ${ }^{1}$ Department of Informatics, Universitas Musamus, Merauke 99600, Indonesia. \\ ${ }^{2}$ Department of Animal Husbandry, Universitas Musamus, Merauke 99600, Indonesia
}

\begin{abstract}
Merauke is one of seventeen districts selected for strengthening beef cattle breeding activities to provide livestock germs, especially for local livestock, through domestic procurement. The sustainability of domestic beef production supplies must be supported by the availability of quality beef breeds. Superior beef cattle are one of the determining factors and have a strategic value in the effort to develop beef cattle. Criteria for evaluating the quality of cattle breeds, including body size or morphology, health and defects in livestock, are in accordance with Indonesian national standards for good beef cattle breeding guidelines. Selection of cattle breeds is done by the traditional breeders community only by visually paying attention to the size of cattle. This study aims to develop decision support tools for farmers in selecting prospective highquality beef cattle, to analyze the criteria data for cattle using a combination of two methods; AHP and SMART, that alternative solutions can be obtained that will be recommended to farmers. The local beef cattle of Merauke that are used as research samples are PO cattle, system provides information on bull superior which farmers can use as supporting information in making decisions.
\end{abstract}

Keywords: Decision Support System, Hybrid, AHP, SMART

\section{Introduction}

Beef consumption is estimated to rise by $6.01 \%$, while production is only up by $2.5 \%$, causing meat prices to rise sharply to $19.4 \%$. Increased population growth has an impact on the total national consumption of beef is also increasing and is an opportunity for the national beef cattle industry to be able to fulfill it [1]. Merauke is a district in Papua Province which is to participate in supporting self-sufficiency in beef, in strengthening beef cattle breeding activities to provide livestock germs, especially for native or local livestock, through domestic procurement [2]. Provision of beef production and to ensure its sustainability, it is necessary to have a continuous supply of beef cattle. Livestock germs are animals that have characteristics and inherit them and fulfill certain requirements to be bred with good reproductive performance [3].

The process of selecting superior breeding cattle is based on the performance of the child and individual prospective beef cattle breeding using certain selection criteria [4]. Assessment of the quality of cattle breeds can be seen from various aspects, including body size or morphology, health of cattle from all types of diseases, and physical defects of cattle in accordance with Indonesian national standards in good beef breeding guidelines. However, the choice of cattle breeders is traditionally done by visually paying attention to the size of cattle. Decision support application is an alternative that aims to assist farmers [5] in choosing prospective high-quality beef cattle. The method used to analyze the criteria for the selection of superior beef cattle using a combination AHP and SMART. Multi criteria decision making method with AHP, is used in determining products that have prospective value so that it can be developed in an integrated manner [6]. Application of decision support systems in risk management for the development of palm oil-based biodiesel agroindustry, with analysis and interpretation models using AHP [7]. Development of intelligent systems with decisionmaking methods [8]. Comparison of the performance of the AHP and TOPSIS methods, shows that AHP method produces a different alternative ranking [9]. SMART is a method for multi criteria data analysis, which means that each alternative has a criterion and weight, each criterion weight that illustrates how important one criterion is with other criteria[10]. Examples of the adoption of a decision support system to recommend the application of appropriate dosage fertilizers, which form the basis of evidence-based decision making in agriculture [11][12].

Local cattle breed used as research samples are Ongole Peranakan cattle (PO), which are local Indonesian beef cattle family, which have characteristics of physical shape and genetic

\footnotetext{
* Corresponding author : lilik@unmus.ac.id
} 
composition and ability to adapt to various environments [13]. Breeding superior beef cattle will produce offspring that can increase profits and produce meat that has competitiveness with imported products. Conversely, if the selection of beef cattle does not comply with established standards, it will have an impact on the lack of meat production, thus causing high prices for beef.

\section{Material and Methods}

Decision support system application aims to assist farmers in selecting superior breeds of cattle, application will recommend alternatives superior cattle breeds, so the results of the solutions offered can be used for the decision-making process by farmers.

\subsection{Hybrid Algorithm}

Hybrid algorithms, is new approach to the hybrid Multiple-Criteria Decision-Making (MCDM) method has been widely developed, including the decision to review papers that have been distributed according to specific publishing periods. The use of certain methods for data analysis to support decision making, is one of the stages carried out the information for decision makers[14]. The steps to solve the problem of selecting superior breeding cattle with the hybrid method are explained as follows:

1. Determine a list of the main criteria, which are used for the selection of superior cattle breeds.

2. Creating or compiling a hierarchy of objectives, criteria and alternatives (superior breeds of cattle) in accordance with the AHP concept.

3. Determine the importance of each criteria.

4. Make a pairwise comparison matrix in accordance with the importance of the criteria specified in the previous step. Then normalize matrix by dividing each value in the matrix column by the sum of the corresponding columns.

5. Test the consistency ratio (CR) for the hierarchy. If the consistency is less than 0.1 , then the data analysis for selecting superior breeds of cattle is continued by the SMART method.

6. Normalization of the criteria value matrix for each cattle, so that the data to be analyzed is in the same range.

7. Calculate value of utility for alternative (cattle).

8. Sorting the results of data analysis of cattle ranging from the largest utility to the lowest, so that ranking can be determined for each alternative (cattle).

9. Choose cattle that have the greatest utility value.

Stages cattle data analysis by applying hybrid algorithm based on multi-criteria decision making by combining two methods of AHP and SMART, shown in Figure 1.

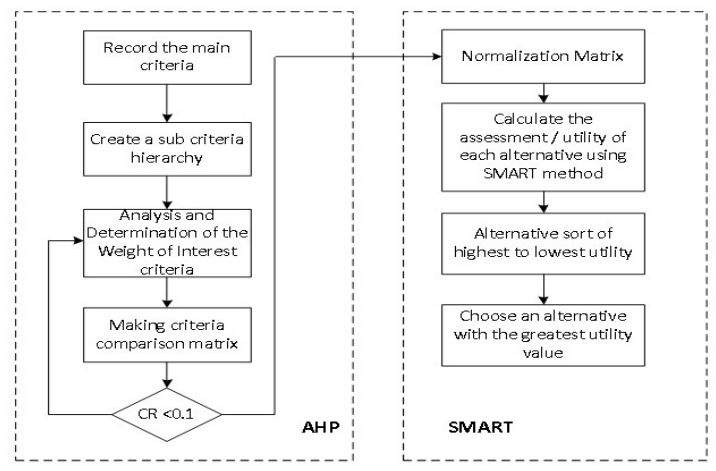

Fig. 1. Hybrid Method flow diagram

\subsection{Analysis of Heirarcy Criteria using AHP method}

Implementation of AHP method using a hierarchy of criteria, alternatives, and objectives, then proceed with determining the weight or priority interests of each criteria in the form of a paired comparison matrix [6][8].

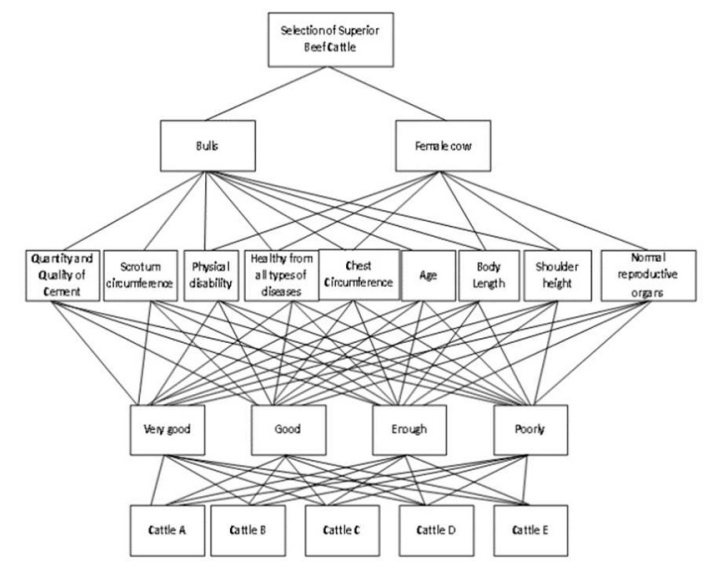

Fig. 2. AHP hierarcy of goal, criterions and alternatives

Figure 2, shows the relationship between the criteria and alternatives using AHP. The next step is, examine the consistency ratio of pairwise comparison ratios to assess alternatives and criteria, with determine priority of criteria and alternative. Table 1 shows the list of criteria for selection of superior bulls.

Table 1. Criteria for selection of superior bull.

\begin{tabular}{|c|c|}
\hline Criteria & Description \\
\hline $\mathrm{SB}_{1}$ & Healthy \\
\hline $\mathrm{SB}_{2}$ & Normal reproductive organs \\
\hline $\mathrm{SB}_{3}$ & Good quality and quantity of cement \\
\hline $\mathrm{SB}_{4}$ & Cattle Age (Months) \\
\hline $\mathrm{SB}_{5}$ & Shoulder Height \\
\hline $\mathrm{SB}_{6}$ & Body length \\
\hline $\mathrm{SB}_{7}$ & Chest Circumference \\
\hline $\mathrm{SB}_{8}$ & The scrotum circumference \\
\hline
\end{tabular}


Determination of each value in the pairwise comparison matrix, using the policy of decision makers or experts, by determining the level of importance of one criterion against other criteria. The assessment process starts at the top level of the hierarchy and determine the value of importance between criteria, based on scale. $x_{i j}=\frac{1}{x_{j i}}$

Description: $\mathrm{x}=$ value matrix, $\mathrm{i}=$ row, $\mathrm{j}=$ columns.

\subsection{SMART Methods}

SMART method is continued by determining the utility for each alternative. The determination of utility begins by converting the criterion value of each alternative using the following equation [15].

$u_{i}\left(a_{i}\right)=\frac{c_{\text {out }}-c_{\min }}{c_{\max }-c_{\min }}$

\section{Result and Discussion}

Table 2 shows the making of a matrix by comparing one criterion with another, and giving importance to each criterion, for the upper triangle matrix based on a scale of 1-9. Whereas the lower triangular matrix uses equation 1 [16].

Table 2. Making pairwise comparison matrices

\begin{tabular}{|c|c|c|c|c|c|c|c|c|}
\hline & $\mathbf{S B}_{\mathbf{1}}$ & $\mathbf{S B}_{\mathbf{2}}$ & $\mathbf{S B}_{\mathbf{3}}$ & $\mathbf{S B}_{\mathbf{4}}$ & $\mathbf{S B}_{\mathbf{5}}$ & $\mathbf{S B}_{\mathbf{6}}$ & $\mathbf{S B}_{\mathbf{7}}$ & $\mathbf{S B}_{\mathbf{8}}$ \\
\hline $\mathrm{SB}_{1}$ & 1.00 & 3.00 & 3.00 & 4.00 & 5.00 & 6.00 & 6.00 & 7.00 \\
\hline $\mathrm{SB}_{2}$ & 0.33 & 1.00 & 3.00 & 4.00 & 5.00 & 5.00 & 5.00 & 5.00 \\
\hline $\mathrm{SB}_{3}$ & 0.33 & 0.33 & 1.00 & 3.00 & 4.00 & 2.00 & 2.00 & 2.00 \\
\hline $\mathrm{SB}_{4}$ & 0.25 & 0.25 & 0.33 & 1.00 & 2.00 & 2.00 & 2.00 & 2.00 \\
\hline $\mathrm{SB}_{5}$ & 0.20 & 0.20 & 0.25 & 0.50 & 1.00 & 2.00 & 2.00 & 2.00 \\
\hline $\mathrm{SB}_{6}$ & 0.17 & 0.20 & 0.50 & 0.50 & 0.50 & 1.00 & 2.00 & 2.00 \\
\hline $\mathrm{SB}_{7}$ & 0.17 & 0.20 & 0.50 & 0.50 & 0.50 & 0.50 & 1.00 & 2.00 \\
\hline $\mathrm{SB}_{8}$ & 0.14 & 0.20 & 0.50 & 0.50 & 0.50 & 0.50 & 0.50 & 1.00 \\
\hline Total & 2.59 & 5.38 & 9.08 & 14.00 & 18.50 & 19.00 & 20.50 & 23.00 \\
\hline
\end{tabular}

The next stage is to carry out the matrix normalization process, so that all criterion data is in the same range of $0-1$. Normalization is done using the following equation [17].

$$
x_{i j}^{*}=\frac{\sum_{i=1}^{n} x_{i j}}{n}
$$

Results normalized pairwise comparison matrix shown in Table 3, the results of the calculation of each column numbered 1 , which indicates that every element of the value of the interests of the criteria have been normalized.
Table 3. Matriks normalization

\begin{tabular}{|c|c|c|c|c|c|c|c|c|}
\hline & $\mathbf{S B}_{\mathbf{1}}$ & $\mathbf{S B}_{\mathbf{2}}$ & $\mathbf{S B}_{3}$ & $\mathbf{S B}_{4}$ & $\mathbf{S B}_{\mathbf{5}}$ & $\mathbf{S B}_{\mathbf{6}}$ & $\mathbf{S B}_{7}$ & $\mathbf{S B}_{\mathbf{8}}$ \\
\hline $\mathrm{SB}_{1}$ & 0.39 & 0.56 & 0.33 & 0.29 & 0.27 & 0.32 & 0.29 & 0.30 \\
\hline $\mathrm{SB}_{2}$ & 0.13 & 0.19 & 0.33 & 0.29 & 0.27 & 0.26 & 0.24 & 0.22 \\
\hline $\mathrm{SB}_{3}$ & 0.13 & 0.06 & 0.11 & 0.21 & 0.22 & 0.11 & 0.10 & 0.09 \\
\hline $\mathrm{SB}_{4}$ & 0.10 & 0.05 & 0.04 & 0.07 & 0.11 & 0.11 & 0.10 & 0.09 \\
\hline $\mathrm{SB}_{5}$ & 0.08 & 0.04 & 0.03 & 0.04 & 0.05 & 0.11 & 0.10 & 0.09 \\
\hline $\mathrm{SB}_{6}$ & 0.06 & 0.04 & 0.06 & 0.04 & 0.03 & 0.05 & 0.10 & 0.09 \\
\hline $\mathrm{SB}_{7}$ & 0.06 & 0.04 & 0.06 & 0.04 & 0.03 & 0.03 & 0.05 & 0.09 \\
\hline $\mathrm{SB}_{8}$ & 0.06 & 0.04 & 0.06 & 0.04 & 0.03 & 0.03 & 0.02 & 0.04 \\
\hline Total & 1.00 & 1.00 & 1.00 & 1.00 & 1.00 & 1.00 & 1.00 & 1.00 \\
\hline
\end{tabular}

Calculation of the priority value of the criteria for selecting superior beef cattle is done to determine the priority of each criterion, by adding up each row in the normalized matrix, then divided by the number of criteria used. Table 4 shows the results of determining priorities for each criterion.

Table 4. Calculation of priority criteria

\begin{tabular}{|c|c|c|}
\hline Criteria & Eigenvector & Priority \\
\hline $\mathrm{SB}_{1}$ & 0.3428 & 1 \\
\hline $\mathrm{SB}_{2}$ & 0.2406 & 2 \\
\hline $\mathrm{SB}_{3}$ & 0.1276 & 3 \\
\hline $\mathrm{SB}_{4}$ & 0.0811 & 4 \\
\hline $\mathrm{SB}_{5}$ & 0.0652 & 5 \\
\hline $\mathrm{SB}_{6}$ & 0.0570 & 6 \\
\hline $\mathrm{SB}_{7}$ & 0.0477 & 7 \\
\hline $\mathrm{SB} 8$ & 0.0380 & 8 \\
\hline
\end{tabular}

The next step is to check the consistency of the index based on the results of the analysis. Measurement of the level of consistency is important to do, so that the decisions obtained have a good consistency value. To find out the consistency of the index, use the following equation [18].

$C I=\frac{\gamma_{\max }-n}{n-1}$

Checking the consistency ratio is performed as the final stage, if the value of consistency ratio $<=0.1$, the final result can be declared true. The analysis using the AHP method must be repeated. Calculation of ratio consistency uses the following equation [19].

$$
C R=\frac{C I}{I R}
$$

Analysts results using the AHP method show that a CR value of $-0,68925$ meets the requirements in CR test for the hierarchy ie if the consistency is less than 0.1 
then the analysis of selection data for superior beef cattle is continued with the SMART method.

Application of the SMART method is carried out after the analysis of the main criteria using the AHP method, the process is to obtain the importance weight (eigen vector) of each criterion. The next step is determining the classification for each sub criteria. An explanation of the sub criteria and classification value for each sub criteria is shown in Table 5 . The sub criteria analysis process, using the SMART method, is by determining the parameter values for each classification of sub criteria. This process begins with melist sub criteria, then determining the classification value.

Table 5. Sub-criteria for selection superior bulls

\begin{tabular}{|c|c|c|}
\hline Criteria & Sub- Criteria & Value \\
\hline \multirow{4}{*}{$\mathrm{SB}_{1}$} & Very good & 4 \\
\hline & Good & 3 \\
\hline & Enough & 2 \\
\hline & Poorly & 1 \\
\hline \multirow{4}{*}{$\mathrm{SB}_{2}$} & Very good & 4 \\
\hline & Good & 3 \\
\hline & Enough & 2 \\
\hline & Poorly & 1 \\
\hline \multirow{4}{*}{$\mathrm{SB}_{3}$} & Very good & 4 \\
\hline & Good & 3 \\
\hline & Enough & 2 \\
\hline & Poorly & 1 \\
\hline \multirow{4}{*}{ SB4 } & $>36$ months & 4 \\
\hline & $25-36$ month & 3 \\
\hline & 18-24 month & 2 \\
\hline & $<18$ month & 1 \\
\hline \multirow{4}{*}{$\mathrm{SB}_{5}$} & $>=133 \mathrm{~cm}$ & 4 \\
\hline & $128-132 \mathrm{~cm}$ & 3 \\
\hline & $122-127 \mathrm{~cm}$ & 2 \\
\hline & $<122 \mathrm{~cm}$ & 1 \\
\hline \multirow{4}{*}{$\mathrm{SB}_{6}$} & $>=139 \mathrm{~cm}$ & 4 \\
\hline & $130-138 \mathrm{~cm}$ & 3 \\
\hline & $124-129 \mathrm{~cm}$ & 2 \\
\hline & $<124 \mathrm{~cm}$ & 1 \\
\hline \multirow{4}{*}{$\mathrm{SB}_{7}$} & $>175 \mathrm{~cm}$ & 4 \\
\hline & $160-175 \mathrm{~cm}$ & 3 \\
\hline & $144-159 \mathrm{~cm}$ & 2 \\
\hline & $<144 \mathrm{~cm}$ & 1 \\
\hline \multirow{4}{*}{$\mathrm{SB}_{8}$} & $>=26 \mathrm{~cm}$ & 4 \\
\hline & $24-25 \mathrm{~cm}$ & 3 \\
\hline & $22-23 \mathrm{~cm}$ & 2 \\
\hline & $<22 \mathrm{~cm}$ & 1 \\
\hline
\end{tabular}

In the following table describes the value of the criteria for each alternative cattle bulls.
Table 6. Alternative yielding bulls and value of criteria

\begin{tabular}{|c|c|c|c|c|c|c|c|c|}
\hline \multirow{2}{*}{$\begin{array}{c}\text { Alternat } \\
\text { ive }\end{array}$} & \multicolumn{7}{|c|}{ Criteria } \\
\cline { 2 - 9 } & $\begin{array}{c}\text { SB } \\
\mathbf{1}\end{array}$ & $\begin{array}{c}\text { SB } \\
\mathbf{2}\end{array}$ & $\begin{array}{c}\text { SB } \\
\mathbf{3}\end{array}$ & $\begin{array}{c}\text { SB } \\
\mathbf{4}\end{array}$ & $\begin{array}{c}\text { SB } \\
\mathbf{5}\end{array}$ & $\begin{array}{c}\text { SB } \\
\mathbf{6}\end{array}$ & $\begin{array}{c}\text { SB } \\
\mathbf{7}\end{array}$ & $\begin{array}{c}\text { SB } \\
\mathbf{8}\end{array}$ \\
\hline $\mathrm{B}_{1}$ & 4 & 4 & 3 & 3 & 3 & 2 & 3 & 2 \\
\hline $\mathrm{B}_{2}$ & 3 & 3 & 4 & 2 & 3 & 3 & 3 & 2 \\
\hline $\mathrm{B}_{3}$ & 4 & 3 & 4 & 3 & 3 & 4 & 3 & 3 \\
\hline $\mathrm{B}_{4}$ & 4 & 4 & 4 & 2 & 3 & 2 & 4 & 2 \\
\hline $\mathrm{B}_{5}$ & 3 & 4 & 3 & 4 & 4 & 4 & 3 & 4 \\
\hline $\mathrm{B}_{6}$ & 4 & 3 & 4 & 4 & 4 & 4 & 3 & 3 \\
\hline $\mathrm{B}_{7}$ & 3 & 3 & 3 & 4 & 4 & 3 & 4 & 3 \\
\hline $\mathrm{B}_{8}$ & 4 & 4 & 3 & 2 & 3 & 3 & 4 & 3 \\
\hline $\mathrm{B}_{9}$ & 4 & 3 & 4 & 3 & 2 & 3 & 4 & 4 \\
\hline $\mathrm{B}_{10}$ & 4 & 4 & 4 & 3 & 4 & 4 & 4 & 3 \\
\hline $\mathrm{B}_{11}$ & 4 & 4 & 3 & 3 & 3 & 4 & 4 & 3 \\
\hline $\mathrm{B}_{12}$ & 4 & 4 & 4 & 3 & 4 & 4 & 4 & 4 \\
\hline $\mathrm{B}_{13}$ & 3 & 4 & 3 & 4 & 4 & 4 & 4 & 4 \\
\hline $\mathrm{B}_{14}$ & 4 & 4 & 4 & 3 & 3 & 4 & 3 & 4 \\
\hline $\mathrm{B}_{15}$ & 4 & 3 & 3 & 4 & 4 & 4 & 3 & 4 \\
\hline
\end{tabular}

Analysis by the SMART method is continued by determining the utility for each alternative. Calculate value of utility by converting the criteria value using the equation [10].

$u_{i}\left(a_{i}\right)=\frac{c_{o u t}-c_{\min }}{c_{\max }-c_{\min }}$

Example of application of equation 5, if the value is for criteria $($ Cout $)=4$, then:

$$
u_{i}\left(a_{i}\right)=\frac{4-1}{4-1}=1
$$

Value for criteria $($ Cout $)=3$, then:

$$
u_{i}\left(a_{i}\right)=\frac{3-1}{4-1}=0.6667
$$

Value for criteria $($ Cout $)=2$, then:

$$
u_{i}\left(a_{i}\right)=\frac{2-1}{4-1}=0.3333
$$

Value for criteria $($ Cout $)=1$, then:

$$
u_{i}\left(a_{i}\right)=\frac{1-1}{4-1}=0
$$

The results of applying SMART Normalization, are shown in table 7 . 
Table 7. Normalization using SMART

\begin{tabular}{|c|c|c|c|c|c|c|c|c|}
\hline \multirow{2}{*}{$\begin{array}{c}\text { Alternati } \\
\text { ve }\end{array}$} & \multicolumn{8}{|c|}{ Criteria } \\
\hline & SB & SB & SB & SB & $\begin{array}{c}\text { SB } \\
5\end{array}$ & $\begin{array}{c}\text { SB } \\
6\end{array}$ & SB & $\begin{array}{c}\text { SB } \\
8\end{array}$ \\
\hline \multirow{2}{*}{$\mathrm{B}_{1}$} & 1.0 & 1.0 & 0.6 & 0.6 & 0.6 & 0.3 & 0.6 & 0.3 \\
\hline & 0 & 0 & 7 & 7 & 7 & 3 & 7 & 3 \\
\hline \multirow{2}{*}{$\mathrm{B}_{2}$} & 0.6 & 0.6 & 1.0 & 0.3 & 0.6 & 0.6 & 0.6 & 0.3 \\
\hline & 7 & 7 & 0 & 3 & 7 & 7 & 7 & 3 \\
\hline \multirow{2}{*}{$\mathrm{B}_{3}$} & 1.0 & 0.6 & 1.0 & 0.6 & 0.6 & 1.0 & 0.6 & 0.6 \\
\hline & 0 & 7 & 0 & 7 & 7 & 0 & 7 & 7 \\
\hline \multirow{2}{*}{$\mathrm{B}_{4}$} & 1.0 & 1.0 & 1.0 & 0.3 & 0.6 & 0.3 & 1.0 & 0.3 \\
\hline & 0 & 0 & 0 & 3 & 7 & 3 & 0 & 3 \\
\hline \multirow{2}{*}{$\mathrm{B}_{5}$} & 0.6 & 1.0 & 0.6 & 1.0 & 1.0 & 1.0 & 0.6 & 1.0 \\
\hline & 7 & 0 & 7 & 0 & 0 & 0 & 7 & 0 \\
\hline \multirow{2}{*}{$\mathrm{B}_{6}$} & 1.0 & 0.6 & 1.0 & 1.0 & 1.0 & 1.0 & 0.6 & 0.6 \\
\hline & 0 & 7 & 0 & 0 & 0 & 0 & 7 & 7 \\
\hline \multirow{2}{*}{$\mathrm{B}_{7}$} & 0.6 & 0.6 & 0.6 & 1.0 & 1.0 & 0.6 & 1.0 & 0.6 \\
\hline & 7 & 7 & 7 & 0 & 0 & 7 & 0 & 7 \\
\hline \multirow{2}{*}{$\mathrm{B}_{8}$} & 1.0 & 1.0 & 0.6 & 0.3 & 0.6 & 0.6 & 1.0 & 0.6 \\
\hline & 0 & 0 & 7 & 3 & 7 & 7 & 0 & 7 \\
\hline \multirow{2}{*}{$\mathrm{B}_{9}$} & 1.0 & 0.6 & 1.0 & 0.6 & 0.3 & 0.6 & 1.0 & 1.0 \\
\hline & 0 & 7 & 0 & 7 & 3 & 7 & 0 & 0 \\
\hline \multirow{2}{*}{$\mathrm{B}_{10}$} & 1.0 & 1.0 & 1.0 & 0.6 & 1.0 & 1.0 & 1.0 & 0.6 \\
\hline & 0 & 0 & 0 & 7 & 0 & 0 & 0 & 7 \\
\hline \multirow{2}{*}{$\mathrm{B}_{11}$} & 1.0 & 1.0 & 0.6 & 0.6 & 0.6 & 1.0 & 1.0 & 0.6 \\
\hline & 0 & 0 & 7 & 7 & 7 & 0 & 0 & 7 \\
\hline \multirow{2}{*}{$\mathrm{B}_{12}$} & 1.0 & 1.0 & 1.0 & 0.6 & 1.0 & 1.0 & 1.0 & 1.0 \\
\hline & 0 & 0 & 0 & 7 & 0 & 0 & 0 & 0 \\
\hline \multirow{2}{*}{$\mathrm{B}_{13}$} & 0.6 & 1.0 & 0.6 & 1.0 & 1.0 & 1.0 & 1.0 & 1.0 \\
\hline & 7 & 0 & 7 & 0 & 0 & 0 & 0 & 0 \\
\hline \multirow{2}{*}{$\mathrm{B}_{14}$} & 1.0 & 1.0 & 1.0 & 0.6 & 0.6 & 1.0 & 0.6 & 1.0 \\
\hline & 0 & 0 & 0 & 7 & 7 & 0 & 7 & 0 \\
\hline \multirow{2}{*}{$\mathrm{B}_{15}$} & 1.0 & 0.6 & 0.6 & 1.0 & 1.0 & 1.0 & 0.6 & 1.0 \\
\hline & 0 & 7 & 7 & 0 & 0 & 0 & 7 & 0 \\
\hline
\end{tabular}

The calculation of utility values for all alternative bulls are shown in Table 7. This process is carried out to obtain the final value of the analysis of the superior seeds of bull using SMART. Utilities for each alternative are obtained using the following equation [20].

$u\left(a_{i}\right)=\sum_{j=1}^{m} w_{j} u_{i}\left(a_{i}\right)$

Utility value shows the end result of the analysis process using the SMART method. The final step is the selection of superior cattle breeds by conducting an alternative sorting process with the largest to the smallest utility.

Table 8. Utility value of alternative

\begin{tabular}{|c|c|c|c|c|c|}
\hline No & Alternative & Criteria & $\begin{array}{c}\text { Value } \\
\text { of } \\
\text { utilities }\end{array}$ & $\begin{array}{c}\text { Weight } \\
\text { AHP }\end{array}$ & $\begin{array}{l}\text { Result } \\
\text { (score) }\end{array}$ \\
\hline \multirow{8}{*}{1} & \multirow{8}{*}{$\mathrm{B}_{1}$} & $\mathrm{SB}_{1}$ & 1.000 & 0.3428 & \multirow{8}{*}{0.829} \\
\hline & & $\mathrm{SB}_{2}$ & 1.000 & 0.2406 & \\
\hline & & $\mathrm{SB}_{3}$ & 0.667 & 0.1276 & \\
\hline & & $\mathrm{SB}_{4}$ & 0.667 & 0.0811 & \\
\hline & & $\mathrm{SB}_{5}$ & 0.667 & 0.0652 & \\
\hline & & $\mathrm{SB}_{6}$ & 0.333 & 0.057 & \\
\hline & & $\mathrm{SB}_{7}$ & 0.667 & 0.0477 & \\
\hline & & $\mathrm{SB}_{8}$ & 0.333 & 0.038 & \\
\hline \multirow{6}{*}{2} & \multirow{6}{*}{$\mathrm{B}_{2}$} & $\mathrm{SB}_{1}$ & 0.667 & 0.3428 & \multirow{6}{*}{0.670} \\
\hline & & $\mathrm{SB}_{2}$ & 0.667 & 0.2406 & \\
\hline & & $\mathrm{SB}_{3}$ & 1.000 & 0.1276 & \\
\hline & & $\mathrm{SB}_{4}$ & 0.333 & 0.0811 & \\
\hline & & $\mathrm{SB}_{5}$ & 0.667 & 0.0652 & \\
\hline & & $\mathrm{SB}_{6}$ & 0.667 & 0.057 & \\
\hline
\end{tabular}

\begin{tabular}{|c|c|c|c|c|c|}
\hline No & Alternative & Criteria & $\begin{array}{c}\text { Value } \\
\text { of } \\
\text { utilities }\end{array}$ & $\begin{array}{c}\text { Weight } \\
\text { AHP }\end{array}$ & $\begin{array}{l}\text { Result } \\
\text { (score) }\end{array}$ \\
\hline & & $\mathrm{SB}_{7}$ & 0.667 & 0.0477 & \\
\hline & & $\mathrm{SB}_{8}$ & 0.333 & 0.038 & \\
\hline \multirow{8}{*}{3} & \multirow{8}{*}{$\mathrm{B}_{3}$} & $\mathrm{SB}_{1}$ & 1.000 & 0.3428 & \multirow{8}{*}{0.842} \\
\hline & & $\mathrm{SB}_{2}$ & 0.667 & 0.2406 & \\
\hline & & $\mathrm{SB}_{3}$ & 1.000 & 0.1276 & \\
\hline & & $\mathrm{SB}_{4}$ & 0.667 & 0.0811 & \\
\hline & & $\mathrm{SB}_{5}$ & 0.667 & 0.0652 & \\
\hline & & $\mathrm{SB}_{6}$ & 1.000 & 0.057 & \\
\hline & & $\mathrm{SB}_{7}$ & 0.667 & 0.0477 & \\
\hline & & $\mathrm{SB}_{8}$ & 0.667 & 0.038 & \\
\hline \multirow{8}{*}{4} & \multirow{8}{*}{$\mathrm{B}_{4}$} & $\mathrm{SB}_{1}$ & 1.000 & 0.3428 & \multirow{8}{*}{0.861} \\
\hline & & $\mathrm{SB}_{2}$ & 1.000 & 0.2406 & \\
\hline & & $\mathrm{SB}_{3}$ & 1.000 & 0.1276 & \\
\hline & & $\mathrm{SB}_{4}$ & 0.333 & 0.0811 & \\
\hline & & $\mathrm{SB}_{5}$ & 0.667 & 0.0652 & \\
\hline & & $\mathrm{SB}_{6}$ & 0.333 & 0.057 & \\
\hline & & $\mathrm{SB}_{7}$ & 1.000 & 0.0477 & \\
\hline & & $\mathrm{SB}_{8}$ & 0.333 & 0.038 & \\
\hline \multirow{8}{*}{5} & \multirow{8}{*}{$\mathrm{B}_{5}$} & $\mathrm{SB}_{1}$ & 0.667 & 0.3428 & \multirow{8}{*}{0.827} \\
\hline & & $\mathrm{SB}_{2}$ & 1.000 & 0.2406 & \\
\hline & & $\mathrm{SB}_{3}$ & 0.667 & 0.1276 & \\
\hline & & $\mathrm{SB}_{4}$ & 1.000 & 0.0811 & \\
\hline & & $\mathrm{SB}_{5}$ & 1.000 & 0.0652 & \\
\hline & & $\mathrm{SB}_{6}$ & 1.000 & 0.057 & \\
\hline & & $\mathrm{SB}_{7}$ & 0.667 & 0.0477 & \\
\hline & & $\mathrm{SB}_{8}$ & 1.000 & 0.038 & \\
\hline \multirow{8}{*}{6} & \multirow{8}{*}{ B6 } & $\mathrm{SB}_{1}$ & 1.000 & 0.3428 & \multirow{8}{*}{0.891} \\
\hline & & $\mathrm{SB}_{2}$ & 0.667 & 0.2406 & \\
\hline & & $\mathrm{SB}_{3}$ & 1.000 & 0.1276 & \\
\hline & & $\mathrm{SB}_{4}$ & 1.000 & 0.0811 & \\
\hline & & $\mathrm{SB}_{5}$ & 1.000 & 0.0652 & \\
\hline & & $\mathrm{SB}_{6}$ & 1.000 & 0.057 & \\
\hline & & $\mathrm{SB}_{7}$ & 0.667 & 0.0477 & \\
\hline & & $\mathrm{SB}_{8}$ & 0.667 & 0.038 & \\
\hline & & $\mathrm{SB}_{1}$ & 0.667 & 0.3428 & \\
\hline & & $\mathrm{SB}_{2}$ & 0.667 & 0.2406 & \\
\hline & & $\mathrm{SB}_{3}$ & 0.667 & 0.1276 & \\
\hline 7 & $\mathrm{P}_{-}$ & $\mathrm{SB}_{4}$ & 1.000 & 0.0811 & 0731 \\
\hline 7 & $\mathrm{~B}_{7}$ & $\mathrm{SB}_{5}$ & 1.000 & 0.0652 & 0.731 \\
\hline & & $\mathrm{SB}_{6}$ & 0.667 & 0.057 & \\
\hline & & $\mathrm{SB}_{7}$ & 1.000 & 0.0477 & \\
\hline & & $\mathrm{SB}_{8}$ & 0.667 & 0.038 & \\
\hline & & $\mathrm{SB}_{1}$ & 1.000 & 0.3428 & \\
\hline & & $\mathrm{SB}_{2}$ & 1.000 & 0.2406 & \\
\hline & & $\mathrm{SB}_{3}$ & 0.667 & 0.1276 & \\
\hline 8 & $\mathrm{~B}_{\mathrm{C}}$ & $\mathrm{SB}_{4}$ & 0.333 & 0.0811 & 0850 \\
\hline & $\mathrm{B}_{8}$ & $\mathrm{SB}_{5}$ & 0.667 & 0.0652 & 0.850 \\
\hline & & $\mathrm{SB}_{6}$ & 0.667 & 0.057 & \\
\hline & & $\mathrm{SB}_{7}$ & 1.000 & 0.0477 & \\
\hline & & $\mathrm{SB}_{8}$ & 0.667 & 0.038 & \\
\hline & & $\mathrm{SB}_{1}$ & 1.000 & 0.3428 & \\
\hline & & $\mathrm{SB}_{2}$ & 0.667 & 0.2406 & \\
\hline & & $\mathrm{SB}_{3}$ & 1.000 & 0.1276 & \\
\hline 0 & $\mathrm{P}$ & $\mathrm{SB}_{4}$ & 0.667 & 0.0811 & 0830 \\
\hline 9 & $\mathrm{~B}_{9}$ & $\mathrm{SB}_{5}$ & 0.333 & 0.0652 & 0.830 \\
\hline & & $\mathrm{SB}_{6}$ & 0.667 & 0.057 & \\
\hline & & $\mathrm{SB}_{7}$ & 1.000 & 0.0477 & \\
\hline & & $\mathrm{SB}_{8}$ & 1.000 & 0.038 & \\
\hline & & $\mathrm{SB}_{1}$ & 1.000 & 0.3428 & \\
\hline & & $\mathrm{SB}_{2}$ & 1.000 & 0.2406 & \\
\hline & & $\mathrm{SB}_{3}$ & 1.000 & 0.1276 & \\
\hline 10 & $\mathrm{P}$ & $\mathrm{SB}_{4}$ & 0.667 & 0.0811 & 0060 \\
\hline 10 & $\mathrm{~B}_{10}$ & $\mathrm{SB}_{5}$ & 1.000 & 0.0652 & 0.960 \\
\hline & & $\mathrm{SB}_{6}$ & 1.000 & 0.057 & \\
\hline & & $\mathrm{SB}_{7}$ & 1.000 & 0.0477 & \\
\hline & & $\mathrm{SB}_{8}$ & 0.667 & 0.038 & \\
\hline 11 & $\mathrm{~B}_{11}$ & $\mathrm{SB}_{1}$ & 1.000 & 0.3428 & 0.896 \\
\hline
\end{tabular}




\begin{tabular}{|c|c|c|c|c|c|}
\hline No & Alternative & Criteria & $\begin{array}{c}\text { Value } \\
\text { of } \\
\text { utilities }\end{array}$ & $\begin{array}{c}\text { Weight } \\
\text { AHP }\end{array}$ & $\begin{array}{l}\text { Result } \\
\text { (score) }\end{array}$ \\
\hline & & $\mathrm{SB}_{2}$ & 1.000 & 0.2406 & \\
\hline & & $\mathrm{SB}_{3}$ & 0.667 & 0.1276 & \\
\hline & & $\mathrm{SB}_{4}$ & 0.667 & 0.0811 & \\
\hline & & $\mathrm{SB}_{5}$ & 0.667 & 0.0652 & \\
\hline & & $\mathrm{SB}_{6}$ & 1.000 & 0.057 & \\
\hline & & $\mathrm{SB}_{7}$ & 1.000 & 0.0477 & \\
\hline & & $\mathrm{SB}_{8}$ & 0.667 & 0.038 & \\
\hline \multirow{9}{*}{12} & \multirow{9}{*}{$\mathrm{B}_{12}$} & $\mathrm{SB}_{1}$ & 1.000 & 0.3428 & \multirow{9}{*}{0.973} \\
\hline & & $\mathrm{SB}_{2}$ & 1.000 & 0.2406 & \\
\hline & & $\mathrm{SB}_{3}$ & 1.000 & 0.1276 & \\
\hline & & $\mathrm{SB}_{4}$ & 0.667 & 0.0811 & \\
\hline & & $\mathrm{SB}_{5}$ & 1.000 & 0.0652 & \\
\hline & & $\mathrm{SB}_{6}$ & 1.000 & 0.057 & \\
\hline & & $\mathrm{SB}_{7}$ & 1.000 & 0.0477 & \\
\hline & & $\mathrm{SB}_{8}$ & 1.000 & 0.038 & \\
\hline & & $\mathrm{SB}_{1}$ & 0.667 & 0.3428 & \\
\hline \multirow{7}{*}{13} & \multirow{7}{*}{$\mathrm{B}_{13}$} & $\mathrm{SB}_{2}$ & 1.000 & 0.2406 & \multirow{7}{*}{0.843} \\
\hline & & $\mathrm{SB}_{3}$ & 0.667 & 0.1276 & \\
\hline & & $\mathrm{SB}_{4}$ & 1.000 & 0.0811 & \\
\hline & & $\mathrm{SB}_{5}$ & 1.000 & 0.0652 & \\
\hline & & $\mathrm{SB}_{6}$ & 1.000 & 0.057 & \\
\hline & & $\mathrm{SB}_{7}$ & 1.000 & 0.0477 & \\
\hline & & $\mathrm{SB}_{8}$ & 1.000 & 0.038 & \\
\hline \multirow{8}{*}{14} & \multirow{8}{*}{$\mathrm{B}_{14}$} & $\mathrm{SB}_{1}$ & 1.000 & 0.3428 & \multirow{8}{*}{0.935} \\
\hline & & $\mathrm{SB}_{2}$ & 1.000 & 0.2406 & \\
\hline & & $\mathrm{SB}_{3}$ & 1.000 & 0.1276 & \\
\hline & & $\mathrm{SB}_{4}$ & 0.667 & 0.0811 & \\
\hline & & $\mathrm{SB}_{5}$ & 0.667 & 0.0652 & \\
\hline & & $\mathrm{SB}_{6}$ & 1.000 & 0.057 & \\
\hline & & $\mathrm{SB}_{7}$ & 0.667 & 0.0477 & \\
\hline & & $\mathrm{SB}_{8}$ & 1.000 & 0.038 & \\
\hline \multirow{8}{*}{15} & \multirow{8}{*}{$\mathrm{B}_{15}$} & $\mathrm{SB}_{1}$ & 1.000 & 0.3428 & \multirow{8}{*}{0.861} \\
\hline & & $\mathrm{SB}_{2}$ & 0.667 & 0.2406 & \\
\hline & & $\mathrm{SB}_{3}$ & 0.667 & 0.1276 & \\
\hline & & $\mathrm{SB}_{4}$ & 1.000 & 0.0811 & \\
\hline & & $\mathrm{SB}_{5}$ & 1.000 & 0.0652 & \\
\hline & & $\mathrm{SB}_{6}$ & 1.000 & 0.057 & \\
\hline & & $\mathrm{SB}_{7}$ & 0.667 & 0.0477 & \\
\hline & & $\mathrm{SB}_{8}$ & 1.000 & 0.038 & \\
\hline
\end{tabular}

Based on the results of the analysis of selection of superior beef breeds shown in Table VII, it can be concluded that the alternative or superior beef cattle to be recommended by the system is alternative $B_{12}$ with a final yield value of 0.973 . As for the second order $\mathrm{B}_{10}$ with the final result 0.960 and the third order $\mathrm{B}_{14}$ with the final value 0.935 .

Methods application for decision making using technology can be used to enrich agricultural potential with the help of computer-based decision support systems in agricultural management [21]. The Decision Support System (DSS) application was developed to assist decision makers in the evidence-based decision making process [7]. The application of two hybrid MCDM methods to determine the smart home alternatives, Fuzzy AHP and Fuzzy TOPSIS, shows the results of alternative similar solutions [13]. The selection of priorities for improving government asset management with data analysis using a hybrid method which is a combination of two methods namely; AHP and TOPSIS, produce tests with an accuracy rate of $83 \%$ [24].

The application of the AHP method has been used for the analysis of health infrastructure site selection
[25]. Development of decision support aids to determine the priority of agricultural land to be conserved [26]. The use of technology, can be used to solve complex problems, like consulting directly with experts [27, 28]. The system intelligently supports decision makers namely software manufacturers to choose the technology they want to develop their products [29]. The selection of hospitals that have the best services and treatments that become the credibility of the hospital, based on several qualitative and quantitative criteria using the integration method of Fuzzy Decision Making Trial and Evaluation (FDEMATEL), Fuzzy Analysis Hierarchy Process (FAHP), and Fuzzy VIKOR (FVIKOR) .Implementation of AHP and SMART methods for the selection of superior beef cattle, shown by the results of alternative recommendations for superior beef cattle and their ranking can be seen in Table 9.

Table 9. Result of Hybrid Method

\begin{tabular}{|c|c|c|}
\hline Alternative & Score & Ranking \\
\hline $\mathrm{B}_{12}$ & 0.973 & 1 \\
\hline $\mathrm{B}_{10}$ & 0.960 & 2 \\
\hline $\mathrm{B}_{14}$ & 0.935 & 3 \\
\hline $\mathrm{B}_{11}$ & 0.896 & 4 \\
\hline $\mathrm{B}_{6}$ & 0.891 & 5 \\
\hline $\mathrm{B}_{15}$ & 0.861 & 6 \\
\hline $\mathrm{B}_{4}$ & 0.861 & 7 \\
\hline $\mathrm{B}_{8}$ & 0.850 & 8 \\
\hline $\mathrm{B}_{13}$ & 0.843 & 9 \\
\hline $\mathrm{B}_{3}$ & 0.842 & 10 \\
\hline $\mathrm{B}_{9}$ & 0.830 & 11 \\
\hline $\mathrm{B}_{1}$ & 0.829 & 12 \\
\hline $\mathrm{B}_{5}$ & 0.827 & 13 \\
\hline $\mathrm{B}_{7}$ & 0.731 & 14 \\
\hline $\mathrm{B}_{2}$ & 0.670 & 15 \\
\hline
\end{tabular}

\section{Conclusion}

The development of a hybrid method for MCDA decision making that combines the AHP and SMART methods, with the case of selecting superior cattle can recommend best alternatives accompanied by ranking for each alternative, which can be used for decision makers as evidence for the selection of cattle breeds. This study describes a combination of two methods for criteria analysis in a decision support system. The analysis process for decision making can be further developed by combining with other methods.

\section{References}

1. Kementerian Perdagangan Republik Indonesia, "Laporan Ringkas Outlook Pangan Daging Sapi 2015-2019," (2015).

2. Badan Litbang Pertanian, Menuju Bibit Ternak Berstandar SNI, 21st-27 Agus ed. Jakarta: AgroinovasI Sinar tani, (2013). 
3. Kementerian Pertanian, Pedoman Pembibitan Sapi Potong yang Baik. Peraturan Menteri Pertanian Republik Indonesia, (2014).

4. L. Sumaryanti and Nurcholis, "Analysis of Multiple Criteria Decision Making Method for Selection the Superior Cattle," INTENSIF J. Ilm. Penelit. dan Penerapan Teknol. Sist. Inf., vol. 4, no. 1, pp. 131-141, (2020).

5. V. Patil, S. Payer, T. Teli, and S. Jaychandran, "Decision Support System for Agriculture Management," Int. J. Emerg. Trends Sci. Technol., vol. 3, no. 2, pp. 3505-3508, (2016).

6. L. Sumaryanti, T. K. Rahayu, A. Prayitno, and Salju, "Comparison study of SMART and AHP method for paddy fertilizer recommendation in decision support system," IOP Conf. Ser. Earth Environ. Sci., vol. 343, no. 1, (2019).

7. C. S. Wang, H. L. Yang, and S. L. Lin, "To Make Good Decision: A Group DSS for Multiple Criteria Alternative Rank and Selection," Math. Probl. Eng., vol. 2015, pp. 1-15, (2015).

8. D. Dalalah, F. Al-oqla, and M. Hayajneh, "Application of the Analytic Hierarchy Process ( AHP ) in Multi- Criteria Analysis of the Selection of Cranes," vol. 4, no. 5, pp. 567-578, (2010).

9. M. Velasquez and P. T. Hester, "An Analysis of Multi-Criteria Decision Making Methods," Int. J. Oper. Res., vol. 10, no. 2, pp. 56-66, (2013).

10. D. Siregar, D. Arisandi, A. Usman, D. Irwan, and R. Rahim, "Research of Simple Multi-Attribute Rating Technique for Decision Support," J. Phys. Conf. Ser., pp. 1-6, (2017).

11. L. Sumaryanti, L. Lamalewa, and T. Istanto, "Implementation of Fuzzy Multiple Criteria Decision Making for Recommendation Paddy Fertilizer," Int. J. Mech. Eng. Technol., vol. 10, no. 3, pp. 236-243, (2019).

12. R. Rupnik, M. Kukar, P. Vra, D. Ko, D. Pevec, and Z. Bosni, "AgroDSS : A decision support system for agriculture and farming," Comput. Electron. Agric., no. April, (2018).

13. E. Kerselaers, E. Rogge, L. Lauwers, and G. Van Huylenbroeck, "Decision support for prioritising of land to be preserved for agriculture: Can participatory tool development help?," Comput. Electron. Agric., vol. 110, pp. 208-220, (2015).

14. H. G. Shakouri and Y. N. Tavassoli, "Expert Systems with Applications Implementation of a hybrid fuzzy system as a decision support process : A FAHP - FMCDM - FIS composition," Expert Syst. with Appl. J., vol. 39, pp. 3682-3691, (2012).

15. Risawandi and R. Robbi, "Study of the Simple Multi-Attribute Rating Technique For Decision Support Study of the Simple Multi-Attribute Rating Technique For Decision Support," IJSRST, vol. 2, no. 6, pp. 491-494, (2016).

16. L. Tan and L. Tan, "Cloud-based Decision Support and Automation Decision Support Decision Support and and Automation Automation Agriculture in Orchards Agriculture in Orchards Agriculture in Orchards for for for Precision Precision Precision," Int. Fed. Autom. Control, vol. 49, no. 16, pp. 330-335, (2016).

17. V. Kumbhar, "A Comprehensive Study of Application of Decision Support System in Agriculture in Indian Context," Int. J. Comput. Appl., vol. 63, no. 14, pp. 6-11, (2013).

18. D. C. Rose et al., "Decision support tools for agriculture: Towards effective design and delivery," Agric. Syst. J., vol. 149, pp. 165-174, (2016).

19. J. Fredrickson, M. Mannino, O. Alqahtani, and F. Banaei-kashani, "Using similarity measures for medical event sequences to predict mortality in trauma patients," Decis. Support Syst., vol. 116, no. October 2018, pp. 35-47, (2019).

20. E. K. Zavadskas, J. Antucheviciene, Z. Turskis, and H. Adeli, "Hybrid multiple-criteria decisionmaking methods: A review of applications in engineering," Sci. Iran., vol. 23, no. 1, pp. 1-20, (2016).

21. Marco Bartolozzi, Pierfrancesco Bellini, Paolo Nesi, Gianni Pantaleo., Luca Santi., A Smart Decision Support System for Smart City, IEEE International Conference on Smart City. DOI 10.1109/SmartCity. (2015).57.

22. Ihsan Kaya., Cengiz Kahraman., A Comparison of Fuzzy Multicriteria Decision Making Methods for Intelligent Building Assessme. Journal Of Civil Engineering and Management. 2014 Volume 20(1): 59-69. Doi: $10.3846 / 13923730.2013 .801906$.

23. J. Febriansyah, R. Gernowo, A. Kusumawardhani, Implementation of AHP and TOPSIS Method to Determine the Priority of Improving the Management of Government's Assets, International Journal of Innovative Research in Advanced Engineering (IJIRAE), Volume 4 (March 2017) Issue 03, pp: 46 - 53.

24. B. Dehe, and D. Bamford, Development, Test And Comparison Of Two Multiple Criteria Decision Analysis (MCDA) Models: A Case Of Healthcare Infrastructure Location, Expert Systems with Applications, 42 (2015), pp: 6717-6727.

25. E. Kerselaers, E. Rogge, L. Lauwers, G. V. Huylenbroeck, Decision support for prioritising of land to be preserved for agriculture: Can participatory tool development help, Computers and Electronics in Agriculture 110 (2015), Elsevier, pp: 208-220.

26. L. Sumaryanti, A. Musdholifah, S. Hartati, "Digital Image Processing and Neural Network," TELKOMNIKA Indonesian Journal of Electrical Engineering, vol. 16, no. 1, pp. 182-190, (2015). 
27. L. Sumaryanti, Analisis Citra Digital untuk Klasifikasi Kualitas Beras, Jurnal Ilmiah Mustek Anim Ha, Vol. 7 No. 2, Agustus (2018), pp : 87101.

28. S. Farshidi, S. Jansen, R. de Jong, and S. Brinkkemper, A Decision Support System for Software Technology Selection, Journal of Decision Systems, 2018, Published by Informa UK Limited, https://doi.org/10.1080/12460125.2018.1464821.

29. S. N. Taati, A. E. Dooki, A Hybrid Method of Fuzzy DEMATEL/AHP/VIKOR Approach to Rank and Select The Best Hospital Nurses Of A Years: A Case Study, Journal of Applied Research on Industrial Engineering, Vol. 4, No. 2 (2017), pp: 116-132. 